\title{
Spinal Subdural Abscess: A Rare Complication of Decubitus Ulcer
}

\author{
Natalia Usoltseva, MD; Rafael Medina-Flores, MD; Ateeq Rehman, MD; Swetha Samji, MD; \\ and Matthew D'Costa, MD
}

\begin{abstract}
Spinal subdural abscess (SSA) is an uncommon entity. The exact incidence is unknown, with very few cases reported in the literature. This condition may result in spinal cord compression, thus constituting a medical and neurosurgical emergency. The pathogenesis of SSA is not welldescribed, and the available knowledge is based on case observations only. There is only one case report that describes direct seeding from decubitus ulcers as a possible mechanism for development of SSA. We report a case of subacute onset of quadriplegia in a male patient, age 55 years, due to spinal cord compression from SSA and superimposed spinal subdural hematoma. The direct seeding from decubitus ulcers is thought to be the cause of infection in our patient. We present this case of SSA to elucidate and review the predisposing factors, pathogenesis, clinical presentation, diagnostic modalities, and treatment regarding management of this rare disorder.
\end{abstract}

Keywords: Abscess/complications; Bacteroides infections/complications; Empyema, subdural/diagnosis/microbiology; Spinal cord injury/complications; Subdural abscess

\author{
Corresponding Author: \\ Natalia Usoltseva, MD \\ Department of Internal Medicine \\ Marshfield Clinic \\ 1000 North Oak Avenue \\ Marshfield, WI 54449 \\ Tel: (7I5) 387-550। \\ Fax: (7I5) 389-5757 \\ Email: nusolts@gmail.com \\ Received: May I, 2013 \\ Revised: August 8,2013 \\ Accepted: September 4, 2013
}

doi: $10.3121 / \mathrm{cmr} .2013 .1174$

\begin{abstract}
A male patient, aged 55 years, presented to the emergency room with complaints of bilateral upper extremity weakness. His past medical history was significant for paraplegia and neurogenic bladder from a spinal cord injury, complicated with decubitus ulcers and end-stage renal disease secondary to type II diabetes mellitus. His past surgical history was significant for posterior spine fusion with Harrington rod instrumentation at the level of T10. He developed subacute onset of bilateral upper extremity weakness and paresthesias on the day of presentation. He reported a 2-day history of neck and back pain that he initially contributed to his bedridden state. The patient denied fever, recent infections, or trauma. His vital signs were unremarkable. Physical examination revealed quadriparesis, absence of deep tendon reflexes, hypoesthesia, and decubitus ulcers (figure 1). He had a normal leukocyte count and elevated C-reactive protein. Blood cultures and cultures from the ulcers were obtained. With the concern of spinal cord compression based on presentation, intravenous corticosteroids and broad spectrum antibiotics were initiated. A magnetic resonance imaging (MRI) scan of the spine (figure 2) revealed large elongated epidural collection posteriorly within the cervical spine, extending into the thoracic spine and inferiorly. Spinal cord compression was prominent in the narrowed areas of disc-osteophyte complexes at the levels of C3-4 and C5-6, where the spinal cord was compressed between these disc-osteophyte complexes and the posterior epidural collection, with some mildly increased signal intensity within the cord. The lumbosacral spine was not well imaged due to artifacts of spinal hardware. Emergent drainage of the collection was indicated, but the patient declined. Per the patient's wishes, palliative care was initiated on the day
\end{abstract}




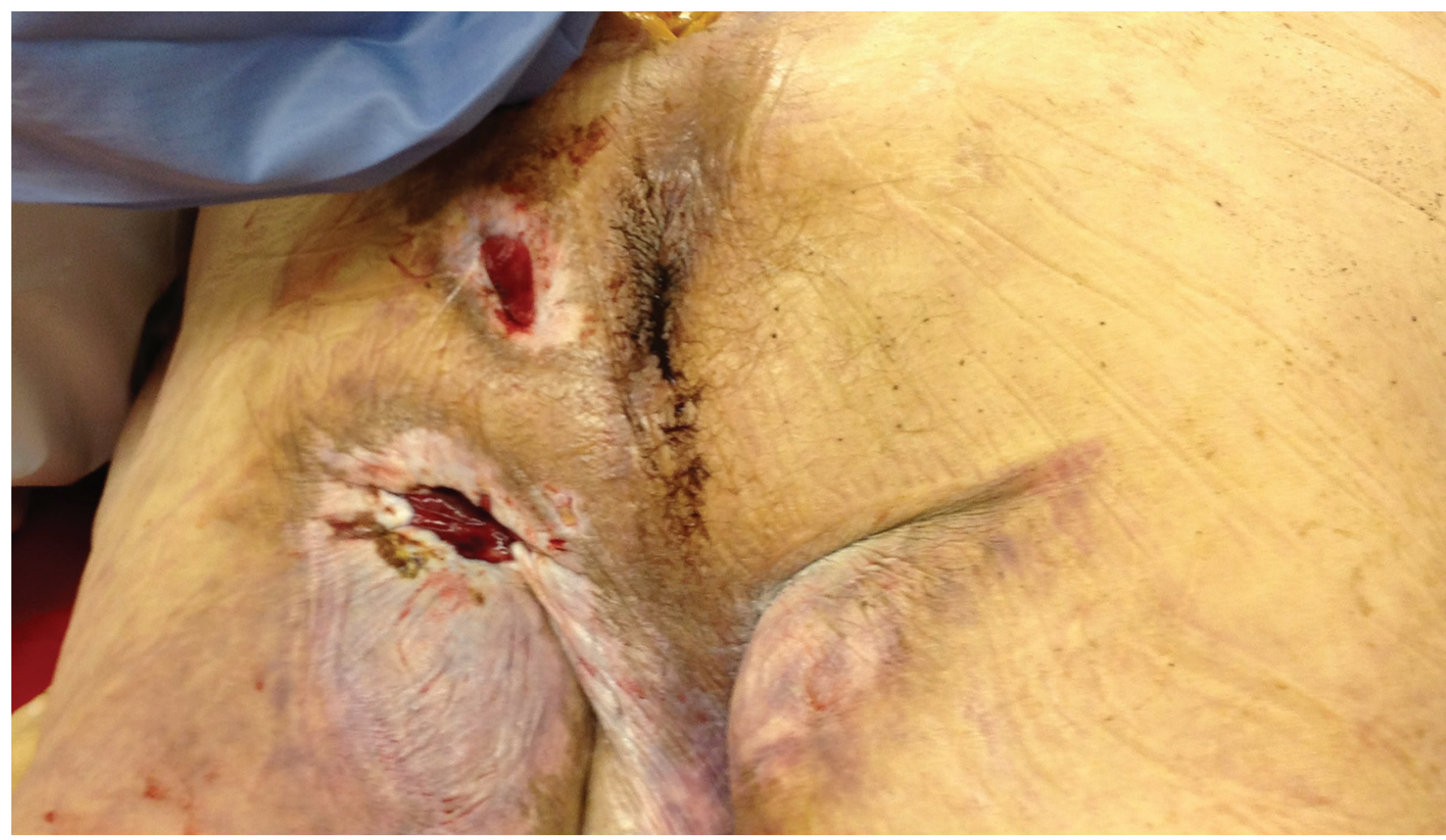

Figure 1: External inspection at the time of autopsy showed multiple Stage III pressure ulcers in the perianal area.

of presentation; all therapy, including antibiotics, was stopped and he died 4 days later.

Autopsy revealed recent hemorrhage in the subdural space (figure 3) extending from the thoracic to the lumbosacral region with spinal cord compression. Purulent exudate (figure 4) was found in the cervical subdural space. Cultures of this purulent material and from decubitus ulcers were identical, growing coagulase-negative staphylococci, Bacteroides fragilis, and Enterobacter cloacae. Blood cultures obtained at admission were negative. Microscopic examination of the spinal cord showed patchy subarachnoid and intraspinal purulent exudate with secondary acute infectious vasculitis causing occlusion of the feeding arterial vessels (figure 5). The cause of sudden onset paraplegia in our patient was due to spinal cord compression secondary to subdural abscess and acute infectious myelitis that caused hemorrhaging in the subdural space.

\section{Discussion}

Spinal subdural abscess (SSA) represents a loculated infection between the outermost layer of the meninges, the dura, and the arachnoid. SSA is rare, and its exact incidence is unknown. ${ }^{1}$ It very uncommonly localizes as a central nervous system infection that may occur secondary to a systemic infection or surgery. ${ }^{1,2}$

There are a few predisposing conditions that contribute to the development of SSA, ${ }^{1,3}$ such as an underlying disease that impairs immunity (diabetes mellitus, alcoholism, tumors, end stage renal disease, hemodialysis, or infection with human immunodeficiency virus), anatomical abnormalities of the spinal cord or vertebral column due to degenerative joint disease, trauma, surgery, drug injection, or placement of catheters. Predisposing conditions for our patient were diabetes mellitus, end stage renal disease, hemodialysis, and a remote history of spinal surgery.

The pathogenesis of SSA is not well described. The available knowledge is based on case observations only. In reviewing the literature, there are three possible mechanisms for development of SSA. ${ }^{4}$

Direct seeding of the infection into the subdural space was reported in association with a thoracic laminectomy complicated by an inadvertent durotomy. ${ }^{5}$ In other reports, subdural empyema was associated with dermal sinus tracts ${ }^{6-8}$ and decubitus ulcers. ${ }^{9}$ The close proximity of ulcers to the sacral dural sac and filum terminale can provide direct anatomical connection to the subdural space. In our patient, we suspect undetected occult direct seeding was confirmed by identical microbiological composition.

Another mechanism might be direct extension from the epidural space where subdural empyema is associated with dura perforation. ${ }^{4}$ This has been reported following epidural catheter insertion, discography, and lumbar puncture. ${ }^{10,11}$ Infection can spread contiguously from the epidural space to the subdural region and can cause subdural empyema associated with cranial epidural abscess. ${ }^{10} \mathrm{~A}$ case of extension 

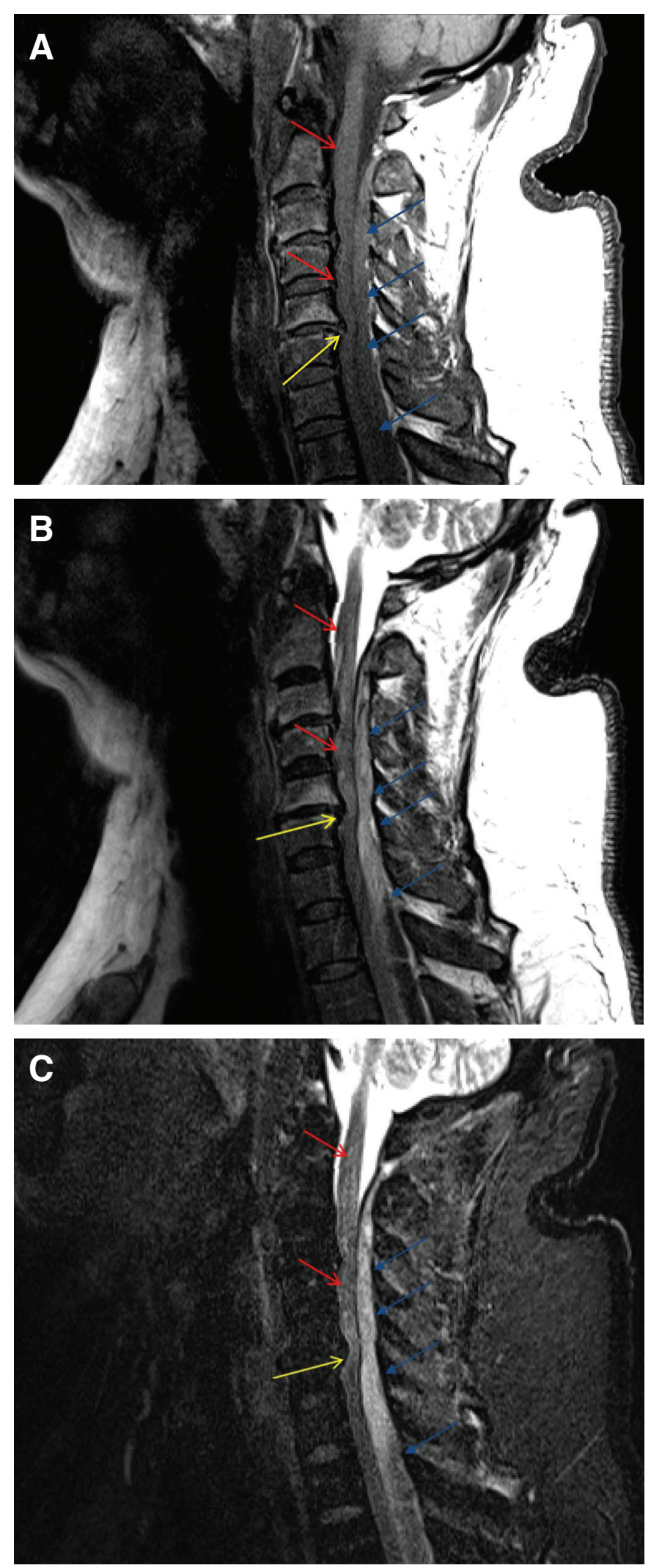

Figure 2: (a) Sagittal T1 Cervical SpineT2, (b) Sagittal T2 Cervical Spine, and (c) Sagittal T2 Fat suppressed image of the cervical spine. Magnetic resonance images demonstrate the posterior epidural collection (blue arrows), spinal cord (red arrows), and region of greatest compression of the spinal cord due to a combination of the posterior epidural collection and the disc-osteophyte complex (yellow arrow) at C5-6. of a retropharyngeal abscess into the subdural space has been described. ${ }^{12}$

The third likely mechanism for the spread of bacteria is hematogenous. ${ }^{4}$ Subdural empyema has been reported after cervical acupuncture ${ }^{13}$ and following meningitis. ${ }^{4}$ In our patient negative blood cultures that were obtained before starting antibiotics and lack of systemic clinical signs of bacteremia make hematogenous spread unlikely. Based on his decision to proceed with palliative care, antibiotics were stopped after he had received only one dose. There are only two cases of SSA in the literature that are unrelated to such conditions and report no well-documented etiology. ${ }^{2}$

The thoracolumbar spine is usually the most affected region, but SSA can involve the entire spine. The typical clinical presentation includes back pain, fever, and neurologic manifestations such as para/tetra paresis, bladder dysfunction, impaired rectal tone, and disturbances of consciousness. ${ }^{2,14-17}$

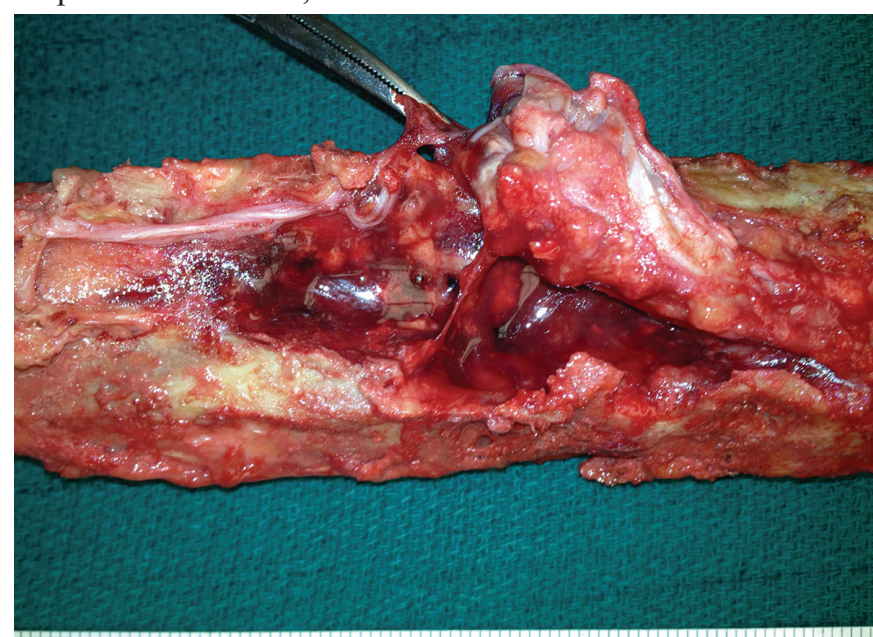

Figure 3: Recent subdural hemorrhage was present surrounding the entire length of the spinal cord at the time of autopsy. The image shows the spinal cord at the level of the conus medullaris; the cauda equina has been removed to mobilize the lower spinal cord.

There are three stages of symptom and physical finding progression: stage one, associated with fever and nerve root pain; stage two manifests with mild neurological deficits; stage three represents the full clinical picture of spinal cord compression such as paralysis and complete sensory loss. ${ }^{15}$ Clinical presentation of SSA may have a typical progression from stage one to three or be presented at any stage. ${ }^{15}$ Our patient had an indolent progression of symptoms over the period of a few days but presented at stage three.

The most powerful modalities for diagnosis of SSA are computerized tomography (CT), MRI, and myeloCT. Contrast-enhanced MRI is superior in detecting the exact location, extension of the abscess, and in diagnosing spinal cord compression. ${ }^{11,15-17}$ Although MRI is the most powerful diagnostic tool, SSA is a surgical diagnosis, because it may be 


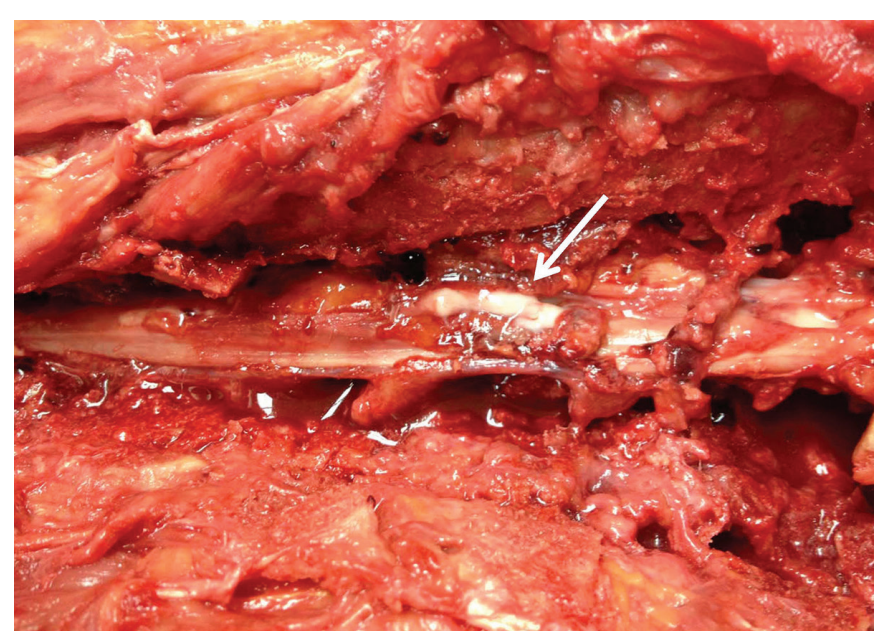

Figure 4: Cervical cord exposed via posterior dissection at the time of autopsy. Arrow points to purulent exudate on the subdural space, note surrounding hemorrhage.

difficult to correctly differentiate epidural from subdural infection. Based on a literature review, there are two case reports where radiographic findings lagged behind the clinical presentation. Those cases described the same discrepancy between the MRI findings of epidural collection and autopsy findings of subdural collection that we observed in our patient. ${ }^{2,4}$ Inflammatory markers such as erythrocyte sedimentation rate and C-reactive protein, as well as leukocyte count can be in the normal range, making them not sensitive indicators of spinal infections. ${ }^{3,9,15}$

SSA may cause rapid compression of the spinal cord and represents an extreme medical and neurosurgical emergency. Prompt surgical drainage and antibiotic therapy are the treatments of choice. The most common causative agents described in the literature are Staphylococcus aureus and anaerobic microaerophilic streptococci. ${ }^{9,10,15,18,19}$ In a majority of cases the causative pathogen is unknown. Empirical choice of antibiotics should include naficillin, oxicillin, or vancomycin plus a cephalosporin and metronidazole. ${ }^{19}$ But, as emphasized by our case, clinical judgment toward possible etiology of SSA should direct the choice of antibiotics.

Surgical drainage is a cornerstone of treatment in a patient with SSA. Neurological impairment can be extremely rapid within full paralyses occurring a few hours after the onset of neurologic deficit. The procedure of choice is laminectomy with debridement of infected tissues. It represents a truly neurosurgical emergency and should be done as soon as possible. ${ }^{1,12}$ Depending on the extension of the abscess, multilevel laminectomy could be necessary, which could cause spinal instability and is associated with poor outcome..$^{20}$ Morbidity and mortality in SSA is high and correlates directly with the delay of the initiation of therapy. ${ }^{9}$ Based on the literature review, there are only few patients with complete

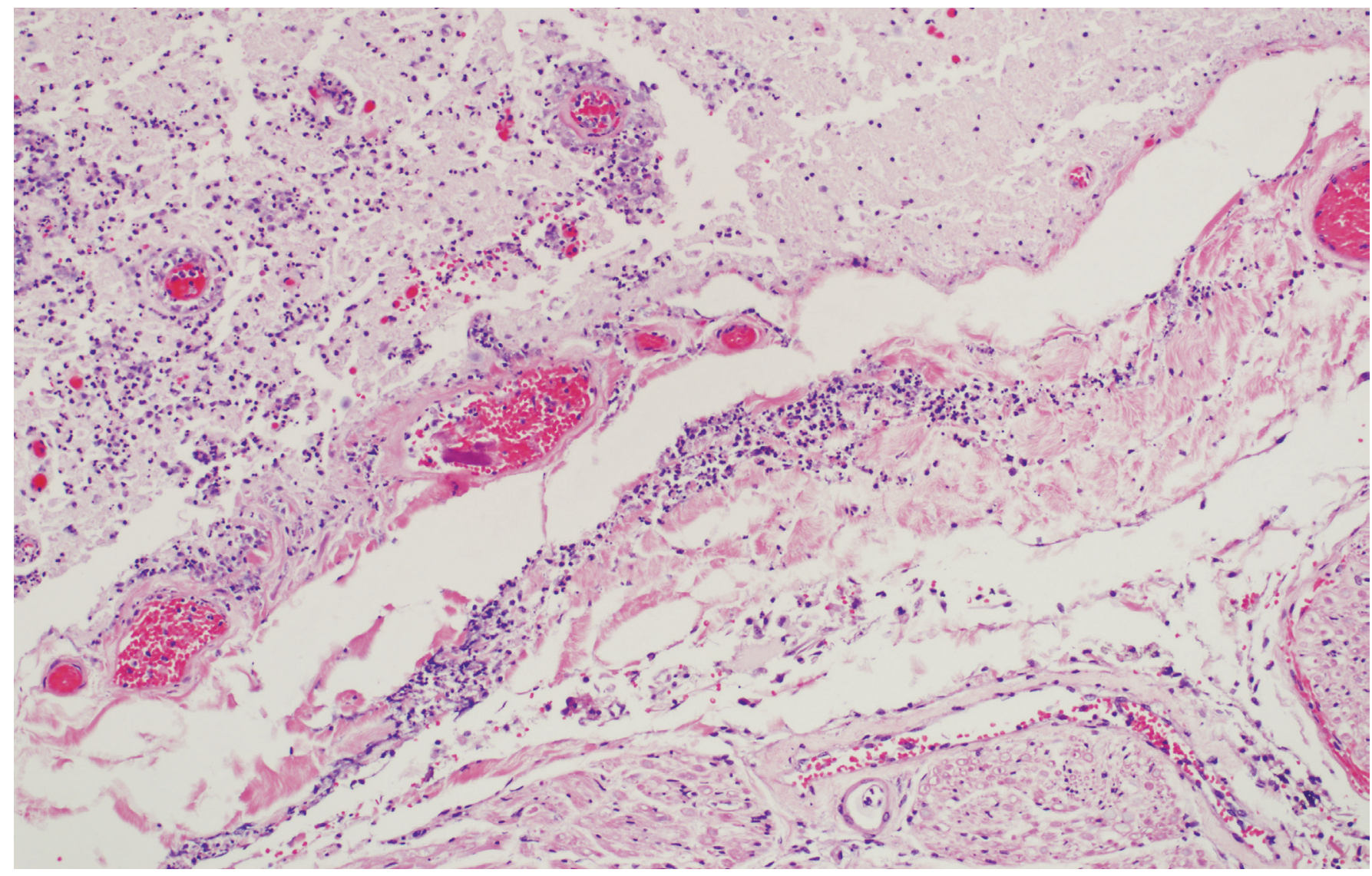

Figure 5: Microsection of the cervical spinal cord with purulent exudate in the subarachnoid space, pial membrane and spinal white matter. Neutrophils dissect the perivascular spaces and partially occlude the lumina of small arteries. (Hematoxylin and eosin stain, 40x magnification.) 
recovery after proper treatment of SSA. ${ }^{2,4}$ The majority of reported cases had a poor neurological outcome or death, even with appropriate therapy and surgical intervention.

The spinal subdural hematoma in our patient was a superimposed finding. The predisposing factors were thought to be due to uremic platelet dysfunction and the use of heparin during hemodialysis in a setting of acute infectious vasculitis. ${ }^{21}$ Both, subdural hematoma and abscess led to spinal cord compression that presented as paraplegia, progressed rapidly, and caused death in our patient.

\section{Conclusion}

Spinal subdural abscess caused by decubitus ulcer is a very rare entity that can lead to superimposed infectious myelitis, hemorrhage, and spinal cord compression. It is associated with high morbidity and mortality. Clinical symptoms such a fever, back pain, and neurological deficits are suggestive of SSA. Etiology and pathogenesis may be uncertain, so a high index of suspicion is needed. First, urgent radiological examination should be obtained; MRI is the investigation of choice, although SSA is a surgical diagnosis. SSA is a neurosurgical emergency, and as soon as diagnosis is established, surgical treatment in collaboration with antibiotic therapy should be performed. Staphylococcus aureus and anaerobic microaerophilic streptococci should be consider as the most possible pathogens, but the etiology should direct the choice of antibiotics. Laminectomy with the debridement of infected tissue is a surgical approach that should be done as soon as possible. Patients who present with prominent neurological deficit and extensive progression might not reverse, despite surgery. Decisions regarding the treatment plan should be done in collaboration with the patient and based on extent of the progression, comorbidities, possible outcomes, and the patient's wishes. Finally, we believe that, despite being rare, decubitus ulcers should be considered as a possible source of subdural space infection.

\section{Acknowledgements}

The authors thank the Marshfield Clinic Research Foundation's Office of Scientific Writing and Publication for editorial assistance in the preparation of this report.

\section{References}

1. Bartels RH, de Jong TR, Grotenhuis JA. Spinal subdural abscess. Case report. J Neurosurg 1992;76:307-311.

2. Sorar M, Er U, Seckin H, Ozturk MH, Bavbek M. Spinal subdural abscess: a rare cause of low back pain. J Clin Neurosci 2008;15:292-294.

3. Hlavin ML, Kaminski HJ, Ross JS, Ganz E. Spinal epidural abscess: a ten year perspective. Neurosurgery 1990;27: 177-184.

4. Coumans JV, Walcott BP. Rapidly progressive lumbar subdural empyema following acromial bursal injection. J Clin Neurosci 2011;18:1562-1563.

5. Wu AS, Griebel RW, Meguro K, Fourney DR. Spinal subdural empyema after a dural tear. Case report. Neurosurg Focus 2004;17:E10.
6. Chen CY, Lin KL, Wang HS, Lui TN. Dermoid cyst with dermal sinus tract complicated with spinal subdural abscess. Pediatr Neurol. 1999;20:157-160.

7. Park SW, Yoon SH, Cho KH, Shin YS, Ahn YH. Infantile lumbosacral spinal subdural abscess with sacral dermal sinus tract. Spine (Phila Pa 1976) 2007;32:E52-E55.

8. Benzil DL, Epstein MH, Knuckey NW. Intramedullary epidermoid associated with an intramedullary spinal abscess secondary to a dermal sinus. Neurosurgery 1992;30:118-121.

9. Baker RP, Brown EM, Coakham HB. Overwhelming cranial and spinal subdural empyema secondary infected sacral decubitus ulcers. Br J Neurosurg 2003;17:572-573.

10. Pompucci A, De Bonis P, Sabatino G, Federico G, Moschini M, Anile C, Mangiola A. Cranio-spinal subdural empyema due to S. intermedius: a case report. J Neuroimaging 2007; 17:358-360.

11. Volk T, Hebecker R, Ruecker G, Perka C, Haas N, Spies C. Subdural empyema combined with paraspinal abscess after epidural catheter insertion. Anesth Analg 2005;100:12221223.

12. Chern SH, Wei CP, Hsieh RL, Wang JL. Methicillin-resistant Staphylococcus aureus retropharyngeal abscess complicated by a cervical spinal subdural empyema. J Clin Neurosci 2009;16:144-146.

13. Chen MH, Chen MH, Huang JS. Cervical subdural empyema following acupuncture. J Clin Neurosci 2004;11:909-911.

14. Gelfand MS, Bakhtian BJ, Simmons BP. Spinal sepsis due to Streptococcus milleri: two cases and review. Rev Infect Dis 1991;13:559-563.

15. Velissaris D, Aretha D, Fligou F, Filos KS. Spinal Subdural Staphylococcus Aureus Abscess: case report and review of the literature. World J Emerg Surg 2009;4:31.

16. Ko MW, Osborne B, Jung S, Jacobs DA, Marcotte P, Galetta SL. Papilledema as a manifestation of a spinal subdural abscess. J Neurol Sci 2007;260:288-292.

17. Carey ME. Infections of the spine and spinal cord. In: Youmans JR, editor. Youmans Neurological Surgery. 4. Philadelphia: WB Saunders; 1996. pp. 3278-3279.

18. Thome C, Krauss JK, Zevgaridis D, Schmiedek P. Pyogenic abscess of the filum terminale. Case report. J Neurosurg 2001;95:100-104.

19. Greenlee JE. Subdural empyema. Curr Treat Options Neurol 2003;5:13-22.

20. Abramovitz JN, Baston RA, Yablon JS. Vertebral osteomyelitis. The surgical management of neurologic complications. Spine (Phila Pa 1976) 1986;11:418-420.

21. Deger SM, Emmez H, Bahadirli K, Kale A, Ebinc FA, Turkoglu M, Arinsoy T, Sindel S. A spontaneous spinal epidural hematoma in a hemodialysis patient: a rare entity. Intern Med 2009;48:2115-2118.

\section{Author Affiliations}

Natalia Usoltseva, MD*; Rafael Medina-Flores, $M D^{\prime}$; Ateeq Rehman, MD*; Swetha Samji, MD*; and Matthew D'Costa, MD*

"Department of Internal Medicine, Marshfield Clinic, Marshfield, Wisconsin, USA

${ }^{\dagger}$ Lab-Pathology, Marshfield Clinic, Marshfield, Wisconsin, USA

${ }^{*}$ Hospital Medicine, Marshfield Clinic, Marshfield, Wisconsin, USA 